\title{
Effect and Associated Factors of a Clinical Pharmacy Model in the Incidence of Medication Errors in the Hospital Pablo Tobón Uribe Eacpharmodel Study: Stepped Wedge Randomized Controlled Trial (NCT03338725).
}

Johan Granados ( $\nabla$ elkyn.granados@udea.edu.co )

Universidad de Antioquia https://orcid.org/0000-0002-3851-2752

\section{Pedro Amariles}

Universidad de Antioquia

Juan Pablo Botero-Aguirre

Hospital Pablo Tobon Uribe

Natalia Andrea Ortiz-Cano

Hospital Pablo Tobon Uribe

Andrés-Felipe Valencia-Quintero

Universidad de Antioquia

Andrea Salazar-Ospina

Universidad de Antioquia

\section{Research Article}

Keywords: Clinical Pharmacy Model, Medication Errors, Pablo Tobón Uribe Eacpharmodel, Stepped Wedge, World Health Organization

Posted Date: March 10th, 2021

DOI: https://doi.org/10.21203/rs.3.rs-268016/v1

License: (c) (i) This work is licensed under a Creative Commons Attribution 4.0 International License. Read Full License

Version of Record: A version of this preprint was published at Trials on January 6th, 2020. See the published version at https://doi.org/10.1186/s13063-019-3945-8. 


\section{Abstract}

\section{Background}

The World Health Organization considers medication errors to be an issue that requires attention at all levels of care, to reduce the severe and preventable harm related to drug therapy. In the world, clinical pharmacy practice standards have been proposed, where the pharmacist, as part of the multidisciplinary health team, can help improve patient safety; however, more and better evidence needs to demonstrate this effect.

\section{Objective}

To assess the change in the incidence of medication errors in hospitalized patients and thereby identify drug-related factors and problems

\section{Setting}

The study was conducted at Hospital Pablo Tobón Uribe, medellín, Colombia.

\section{Methods}

A randomized, controlled cluster-wedge staggered trial with a duration of 14 months was conducted to compare the clinical pharmacy practice model's effect with the usual care process in the hospital. Five hospital health care units were included, which were initially assigned to the control group, and after an observation period of 2 months, they were randomly assigned to the intervention group.

\section{Main outcome measure}

Incidence of medication errors in hospitalized patients

\section{Results}

The medication error incidence was $13.3 \%$ and $22.8 \%$ for the intervention group and control group, respectively. The probability of presenting medication error was $48 \%$ lower when the patient was in the intervention group (RR 0.52; 95\% Cl:0.34-0.79).

\section{Conclusion}

The Clinical pharmacy practice model of the Hospital Pablo Tobón Uribe Hospital, made up of strategies focused on reducing medication errors, significantly reduces medication errors in patients during hospitalization compared to usual practice.

\section{Background}


A medication error (ME) is defined as "any preventable event that may cause or lead to inappropriate medication use or patient harm while the medication is under the control of the health care professional, patient, or consumer." These errors may be associated with any phase of the drug delivery process, from prescription to drug administration[1]. ME represent a severe public health problem that has implications for patient safety and contribute to a considerable increase in the costs of health care and is considered by the World Health Organization (WHO) to be an issue that requires attention at all levels of care, to reduce the severe and preventable harm related to drug therapy. [2-5]

In the hospital setting, approximately $4 \%$ of inpatients are at risk of suffering some damage caused by health care errors or adverse events, of which $70 \%$ may generate temporary disability and $14 \%$ fatal incidents, causing between 44,000 and 98,000 deaths each. [6, 7] At about $37 \%$ of adverse events that occur during the health care process are related to ME Different strategies have been proposed as a global initiative to reduce ME, including implementing clinical pharmacy services. Direct patient care by clinical pharmacists within multidisciplinary teams is recognized as one of the best pharmacy practice models because it minimizes ME, care costs, morbidity and mortality rates, time spent in the hospital, and improved pharmacotherapy results. [8, 9]

In mid-2016, the Hospital Pablo Tobón Uribe (HPTU), a tertiary university hospital located in Medellín (Colombia), standardized a clinical pharmacy practice model (CPPM) that incorporated the activities of the pharmacist into the multidisciplinary health team to provide clinical pharmacy services[10] and to improve patient safety. Currently, this model is actively applied in the hospital; however, knowledge of the model's full effect on patient safety, as framed in the WHO initiative to reduce ME, is needed.[11] The EACPharModel study aims to assess a clinical pharmacy practice model's effectiveness in reducing ME incidence in a tertiary university hospital.

\section{Methods}

This study is based on the EACPHARMODEL trial; a 14-month, randomized, controlled, prospective, singlecenter, Stepped Wedge clinical trial was performed to compare a clinical practice pharmacy model's effect CPPM in the incidence of ME The study began in February 2018 and ended in March 2020. Detailed methods of the EACPHARMODEL trial have been previously published,[12] and the trial has been registered at ClinicalTrials.gov

\section{Design}

Stepped wedge randomized trial designs involved sequential roll-out of an intervention to participants over several periods. At the end of the study, all clusters have received the intervention, the order in which participants receivtae the intervention was determined at random. The clusters were hospital units.

Each medical hospital unit began with a control period (Baseline S0) and changed to an intervention period following randomization. The study design consisted of 6 consecutive 60-day periods (Figure 1). Each 60-day period, another medical hospital unit changed to an intervention period until all units were in 
the intervention period during the final 60-day period. The randomization carried out by the trial defined the point at which each hospital unit changed from the control to the intervention periods.

\section{Setting}

The study was conducted with hospitalized patients prescribed five or more drugs attended in HPTU, a tertiary care university institution. The hospital has 452 beds; however, for the duration of this study, only 5-Medical Hospitalization Units were used.

\section{Study population}

The patients recruited were evaluated whether each patient met all the inclusion criteria: Patients should be at least 18 years old, should be hospitalized in the HPTU for a minimum of 24 hours, and should have at least five drugs in their pharmacological therapy.

\section{Randomization}

Each cluster was assigned to the intervention group (IG) or the control group (CG) through a computergenerated randomization sequence, using Microsoft Excel ${ }^{\circledR}$ (version 2010, Microsoft $\AA$ Corporation, Redmond Washington).

\section{Blinding}

An external evaluation group was made up of an internist and a pharmacist who evaluated if a ME was presented and whether it was resolved. This evaluation was done once the patient's hospitalization was closed and the patient was discharged. Additionally, the evaluators were blinded, and they could not know if the patients were in the clinical pharmacy model.

\section{Sample size}

The overall incidence of ME of $15 \%$ was taken as a reference value. The sample size calculation was made accepting an alpha error of 0.05 , a beta error of $0.84,5$ clusters, 6 steps, 2 months for each step, 24 participants per cluster, and a coefficient of variation $(k)$ of 0.15 . The number of individuals needed to detect a difference equal to or greater than $10 \%$ was $720 .[13]$

\section{Patient recruitment and group assignment}

There was a pharmacist who was responsible for the recruitment of potential patients to the hospitalization units. A cluster was defined as Medical Hospitalization Units, and two months are considered a step. For step 0 or baseline, no cluster had the intervention; for step 1, a cluster was randomly selected to receive the intervention and continue until the end of the study; at step 2, another cluster was randomly selected to receive the intervention and continue until the end of the study; this continued until step 5 where all clusters had the intervention, and the inclusion of the participants within the cluster was dynamic. 


\section{Outcomes}

Determine the change in the incidence of ME by applying the CPPM; The identification, quantification, and classification of ME

Estimation of the probability that a subject remains without ME and measurement of the time until ME were resolved.

\section{Intervention design}

Intervention group: For patients who are under the CPPM of the hospital, pharmacists performed the following activities: (1) participation in medical rounds; (2) medication review, which consists of reviewing patients' medical records and providing verbal or written follow-up concerning the clinical condition (involves repeated monitoring, with review of all medication orders and documentation of pharmaceutical interventions); (3) identification and management of Adverse Drug Reactions (ADR), which consists of detecting potential ADRs, providing and documenting appropriate follow-up until the ADR has resolved, and reporting ADRs to the national pharmacovigilance program; (4) pharmacological counselling to patients, which involves providing information about the proper use of medications to patients and/or family members during the hospital stay or after discharge; (5) pharmacotherapy validation, in which pharmacists conducted an appropriateness review of medical orders and determine, for example, correct dosage, correct frequency, correct route of administration, correct administration, correct duration of therapy, indicated drugs, contraindicated drugs, lack of treatment, drug-drug interactions, drug-food interactions, therapeutic duplicity and allergies.

Every day, the pharmacist in the morning was provided a list of patients that they must evaluate as specified in activities $2,3,5$, and the others when necessary or when they had patients who required it. The model can be seen in figure 2 .

Control group: The usual patient care process began with a medical evaluation and the respective formulation of pharmacotherapy. Later, the pharmacy technicians verified with a spreadsheet the quantities to be dispensed, allergy detection, and therapeutic duplicities, and finally, the medicine was dispensed (Figure 3).

\section{Data collection}

Data was being collected from February 2018 to January 2019. The study had a total length of 14 months, the recruitment period was 12 months, and patients were evaluated for two months, starting from the date of their recruitment. Once the enrolment period ended, the final two months were only used to evaluate the latest patients. The data obtained in this study was registered in an electronic database. Data regarding medication history, interviews with the pharmacist, health status, plans of action, and data related to the primary outcome were registered. 
A specially trained clinical pharmacist and an internist were the staff that reviewed the clinical history of each patient at baseline (t0), two months post-baseline (t1), four months post-baseline (t2), six months post-baseline ( $\mathrm{t} 3$ ), eight months post-baseline ( $\mathrm{t} 4$ ) and ten months post-baseline (t5). The staff was an external evaluation group who evaluated if a ME was presented and whether it was resolved. This evaluation was done once the patient's hospitalization was closed and the patient is discharged.

\section{Statistical analysis}

The statistical analyses of the full-analysis set followed the intention-to-treat principle. This dataset included all subjects in the assigned cluster and met all inclusion criteria. Baseline and demographic characteristics were analyzed descriptively (number of valid cases, mean, standard deviation, median, interquartile range, and proportions for qualitative variables). A mixed model evaluated the primary outcome with treatment group and time as fixed effects and clustering structure as a random effect. A significance level set to alpha $=5 \%$ (two-sided) was used to compare proportions. Comparisons for categorical variables were conducted by using the Chi-square test (or the Fisher exact test when appropriate) and for continuous variables by using the Mann-Whitney U test; Relative Risk and 95\% confidence intervals (C.I.s) was estimated as well. Multivariable analyses were performed to explain the association of multiple variables with the factors significantly related to primary outcome: the sociodemographic and clinical variables assessed were: sex, age, social security system, scholarship, weight, height, allergies, caregiver, diagnosis of admission, hospitalization 6 months before, number of services, previous stay in intensive care unit, ADR, colonized patient, hospital stay and number of medications.

\section{Ethics approval and consent to participate}

The trial was carried out in compliance with the protocol and the declaration of Helsinki, following the international conference on Harmonization. The protocol was approved by the Institutional Review Board of the HPTU (2017.050/2017). The study characteristics are such that the data was collected from clinical records, and the proposed intervention does not entail risk of causing biological, psychological, or social damage, with all the clusters, in the end, having this intervention. Informed consent was obtained from all study participants.

\section{Results}

During the estimated period for the inclusion of patients in the study, 765 patients were identified. After verification of inclusion criteria, of the 765 eligible patients, 45 were excluded because they had a hospital stay of fewer than 24 hours.

The initial population understudy of the EACPHARMODEL was made up of 720 patients, of which they were divided into five different clusters and six steps with 24 participants for each cluster-period of time; all the patients finished the study (Fig. 1). 
The patient population presented a mean age of $60.9(S D=20.4)$ years, and a male prevailed of $50.5 \%$. Table 1 shows the sociodemographic characteristics of the population. At the beginning of the study entry, no statistically significant differences were found between CG and the IG 
Table 1

Baseline Sociodemographic Characteristics of the Patients Included in the EACPharmodel Study.

\begin{tabular}{|c|c|c|c|c|c|}
\hline & \multicolumn{2}{|c|}{ CG n (\%) } & \multicolumn{2}{|c|}{ IG n (\%) } & $\mathbf{p}^{*}$ \\
\hline \multicolumn{6}{|c|}{ Demographic characteristics } \\
\hline Age. median (IQR) & 64 & $(29)$ & 63 & (31) & 0.6025 \\
\hline Weight. median (IQR) & 62 & $(21.6)$ & 63 & $(24.5)$ & 0.4038 \\
\hline Height. median (IQR) & 162 & (15) & 164 & (14) & 0.2225 \\
\hline *BMI. median (IQR) & 23.9 & $(6.5)$ & 23.8 & $(8.0)$ & 0.8040 \\
\hline Sex (Woman) & 184 & $(51.1)$ & 172 & $(47.8)$ & 0.4122 \\
\hline \multicolumn{6}{|l|}{ Security regime } \\
\hline Contributory & 240 & $(66.7)$ & 240 & $(66.7)$ & \multirow[t]{4}{*}{0.8043} \\
\hline Prepaid medicine & 14 & $(3.9)$ & 10 & $(2.8)$ & \\
\hline **TAl & 17 & $(4.7)$ & 15 & $(4.2)$ & \\
\hline Subsidized & 89 & $(24.7)$ & 95 & $(26.4)$ & \\
\hline \multicolumn{6}{|l|}{ Scholarship } \\
\hline No studies & 45 & $(15.3)$ & 52 & $(17.1)$ & \multirow[t]{4}{*}{0.7898} \\
\hline Primary & 146 & $(49.5)$ & 137 & $(45.1)$ & \\
\hline High school & 85 & $(28.8)$ & 97 & (31.9) & \\
\hline University & 64 & $(21.7)$ & 70 & $(23.0)$ & \\
\hline \multicolumn{6}{|l|}{ Caregiver } \\
\hline Yes & 322 & $(89.4)$ & 325 & $(90.5)$ & \multirow[t]{2}{*}{0.7186} \\
\hline No & 38 & $(10.6)$ & 34 & $(9.5)$ & \\
\hline \multicolumn{6}{|l|}{ Allergic history } \\
\hline Yes & 117 & $(32.5)$ & 265 & $(73.6)$ & \multirow[t]{2}{*}{0.086} \\
\hline No & 243 & $(67.5)$ & 95 & $(26.4)$ & \\
\hline \multicolumn{6}{|l|}{ Previous stay in ICU } \\
\hline Yes & 74 & $(20.6)$ & 78 & $(21.7)$ & 0.7841 \\
\hline \multicolumn{6}{|c|}{ * $\mathrm{BMI}=$ Body mass index } \\
\hline * * TAl Traffic accide & ssura & & & & \\
\hline
\end{tabular}




\begin{tabular}{|c|c|c|c|c|c|}
\hline \multirow[b]{2}{*}{ No } & \multicolumn{2}{|c|}{ CG n (\%) } & \multicolumn{2}{|c|}{ IG n (\%) } & \multirow[t]{2}{*}{$\mathrm{p}^{*}$} \\
\hline & 286 & $(79.4)$ & 282 & $(78.3)$ & \\
\hline \multicolumn{6}{|c|}{ Patient in isolation. colonized } \\
\hline Yes & 26 & $(7.2)$ & 24 & $(6.7)$ & \multirow[t]{2}{*}{0.8834} \\
\hline No & 334 & $(92.8)$ & 336 & $(93.3)$ & \\
\hline \multicolumn{6}{|c|}{ History of adverse drug reaction } \\
\hline Yes & 19 & $(5.3$ & 15 & $(4.2)$ & \multirow[t]{2}{*}{0.5981} \\
\hline No & 341 & $(94.7$ & 345 & $(95.8)$ & \\
\hline \multicolumn{6}{|c|}{ * $\mathrm{BMI}=$ Body mass index } \\
\hline \multicolumn{6}{|c|}{ ** TAl Traffic accident insurance } \\
\hline
\end{tabular}

During follow-up, there were no losses and without statistically significant differences at the sociodemographic level $(p>0.05)$

\section{Outcomes}

Incidence of medication errors: A total of 130 MEs were detected (82: CG; 48: IG) with statistically significant differences. The incidence of ME was $13.3 \%$ for IG and 22.8 for CG The estimated RR was 0.52 (I.C. $95 \% 0.34$ to 0.79 ), determining that the probability of presenting a ME was $48 \%$ lower when CPPM follows up the patient, In addition to the intervention, a significant association was also found, and a protective factor was a previous stay in the ICU (RR: 0.44 IC 95\% 0.24 to 0.74 ), Conversely, the increase in the number of medications ordered was a risk factor (RR: 1.09 IC95\% 1.02 to 1.15 ).

\section{Classification of medication errors.}

The most frequent ME among groups were those classified as $\mathrm{C}$, that is, those that reached the patient, but did not generate damage with $31 \%$ for the $C G$ and $26 \%$ for the IG, the ME classified as D or B only was presented in the CG (Table 2). 
Table 2

Medication errors detected classified according to their severity by The National Coordinating Council for Medication Error Reporting and Prevention

\begin{tabular}{|lll|}
\hline Classication of medication errors & $\begin{array}{l}\text { Control } \\
\text { group } \\
\text { n(\%) }\end{array}$ & $\begin{array}{l}\text { Intervention } \\
\text { Group n(\%) }\end{array}$ \\
\hline $\begin{array}{l}\text { B-An error occurred but the error did not reach the patient (An "error of } \\
\text { omission" does reach the patient) }\end{array}$ & $\begin{array}{l}24 \\
(14.6)\end{array}$ & $23(24.0)$ \\
$\begin{array}{l}\text { C-An error occurred that reached the patient but did not cause patient harm } \\
52\end{array}$ & $\begin{array}{l}52 \\
(31.7)\end{array}$ & $2(26.0)$ \\
$\begin{array}{l}\text { D-An error occurred that reached the patient and required monitoring to } \\
\text { confirm that it resulted in no harm to the patient and/or required } \\
\text { intervention to preclude harm }\end{array}$ & $0(0.0)$ \\
$\begin{array}{l}\text { E-An error occurred that may have contributed to or resulted in temporary } \\
\text { harm to the patient and required intervention }\end{array}$ & $4(2.4)$ & $0(0.0)$ \\
\hline
\end{tabular}

\section{Medication errors and measurement of the time}

The probability in the time of presenting a ME was $44 \%$ lower in IG $(p=0.0005)$; meanwhile, the probability in the time of presenting the resolution of a ME was 70\% higher in IG $(p=0.0029)$.

The Cox regression results showed that the group variable was statistically significant $(p=0.0005)$, Medication quantity $(p=0.0004)$ and Previous stay in ICU $(p=0.0018)$ and, Consequently, instantaneous risk (HR:0.56, 95\% Cl=0.39-0.81) were less in the IG than in the $C G$.

For the ME recovery in the Cox regression showed only the study group variable was statistically significant $(p=0.0131)$; consequently, instantaneous risk (HR:1.70, $95 \% \mathrm{Cl}=1.12-2.61)$ was higher in the IG than in the CG (Fig. 4).

\section{Differences in-hospital stay}

The hospital stay data did not follow a normal distribution. Therefore, the Mann Whitney U test was performed, and it was found that there are no statistically significant differences between the median length of stay $(p=0.5620)$

\section{Discussion}

The EACPharModel trial assessed a clinical pharmacy model's effect as demonstrated an effect in reducing $M E$ in hospitalized patients $(R R=0.52)$. To our knowledge, this is the first stepped-wedge controlled trial designed to assess the effect of the CPPM on the incidence of ME in hospitalized patients, generating strong evidence. Another stepped-wedge study, measured collaborative pharmaceutical care's impact on preventable ME rates, but their results are not yet available.[14] 
On the other hand, several articles describe pharmacists' participation and role in detecting ME and their intervention. For example, Khalili et al., reported that clinical pharmacists detected 112 MEs $(0.13$ errors/patient). Dosage, choice, use, and drug interactions were the main causes of error in medication processes, respectively.[15] Additionally, Kuo et al., identified $924 \mathrm{ME}$, of which almost half were corrected before the medications reached the patients.[16] However, these studies do not perform a comparison to determine the model's effect in reducing ME incidence.

The results of a meta-analysis, in which the effect of the intervention of the pharmacist in intensive care units (ICU) on the incidence of ME was evaluated, showed that there is no significant beneficial effect of the intervention on general ME; however, the global analysis supported the role of pharmacists in reducing preventable ADEs and prescribing errors.[17] These results contrast with the EACPharmodel; however, the differences in the study population must be borne in mind. Basheti et al evaluated the pharmacist's impact on the reduction of ME and adherence to treatment in outpatients with chronic diseases, showed a significant decrease in the number of ME with a mean of $(1.23 \pm 1.19 p<0.001)$, in the IG versus $(0.29 \pm 1.24, p=0.114)$ CG[18]

Previous stay in intensive care unit was a protective factor for MEs incidence although it is known that in ICUs, patients are most vulnerable to being exposed to MEs due to brings together high-risk patients and interventions in a complex environment[19-23]; nevertheless, in the HPTU, work has been done on the standardization of processes, prescription and administration of medications through the computerized physician order, and remain all-time in the care of the patient.

Polypharmacy is widespread and associated with medication-related harms, including ADR and ME[24] Our results are consistent and reported the increase in the number of medications ordered as a risk factor $(R R=1.09)$, raising the probability of presenting a ME Medication errors lead to an increase in hospitalization duration [25]; nevertheless, in the present study, the stay was similar in both groups, although the incidence of ME was higher in the CG

In general, ME generates an increase in hospital stay,[25-28] so it would be expected that the hospital stay would be longer in the CG; however, in EACPharmodel study, no statistically significant differences were found in the length of stay in both groups $(p=0.5620)$. This finding is similar to other studies in the hospitalization setting, in which no differences were found in the length of stay. ${ }^{33-35} \mathrm{~A}$ possible explanation could be that a high percentage of errors do not reach the patient or do not cause harm (Type A error); In this sense, only 4 type E errors were recorded in the $C G$ in the present study. For their part, the most frequent $\mathrm{ME}$ among the groups were classified as type $\mathrm{C}$ errors (those that reached the patient but did not cause harm: $36.2 \%$ ), followed by type B errors (did not reach the patient: $59.2 \%$ ). These results are similar to those reported by a study carried out in Spain, in which the incidence of ME in the processes of drug use was described, showing that type B errors were the most frequent (84.5\%), followed by type $C$ errors (14.5\%). ${ }^{36}$ Similarly, in a pharmacovigilance study carried out in Colombian hospitals, $9062 \mathrm{ME}$ were found, of which the most frequent were type A (48.06\%), followed by type B (45.69\%) and type C (5.95\%). ${ }^{37}$ As the ME detection mechanism was the evaluation of medical records, it was not possible to 
find errors with classification A that are "circumstances or incidents with the capacity to cause error," since these events are not recorded in the medical record or the management software of clinical risks.

\section{Conclusion}

The CPPM is made up of strategies focused on reducing ME such as a) pharmacovigilance; $b$ ) medication review; c) Trigger tools method; d) drug conciliation and d) health education significantly reduces the incidence of ME in patients during hospitalization. Regarding ME characterization, those classified as $C, D$, and $E$ are more frequent in the $C G$ than the intervention group. The probability over time of presenting a $\mathrm{ME}$ is higher in the $\mathrm{CG}$ than the intervention group. Besides, the probability over time of the ME resolution is higher in the IC than the CG.

\section{Limitations And Potential Bias}

As the information collected will be based on the review of medical records, some sub-registries may be present that will affect ME detection. However, HPTU is an institution accredited by the Joint Commission International, which has made it possible to adequately standardize the process, including the medical records' quality, which is therefore expected to control this bias.

\section{Declarations}

\section{Acknowledgements}

We would like to thank the Clinical Pharmacy Section, Pharmacy Department, Hospital Pablo Tobón Uribe, Medellín, Colombia. The authors express their gratitude to the Department of Pharmacy, the Faculty of Pharmaceutical and Food Sciences and the University of Antioquia for supporting the project, as well as to the pharmacist Yasmith Manosalva and physician Natalia Zapata for their contributions to patient evaluation.

\section{Funding}

The Pharmaceutical Promotion and Prevention Group received financial support from the Committee for Development Research (CODI) and sustainability program (2018-2019), Universidad de Antioquia.

\section{Conflicts of Interest}

The authors declare no conflict of interes

\section{References}

1. National Coordinating Council for Medication Error Reporting and, Prevention (NCC MERP). National Coordinating Council for Medication Error Re-, porting and Prevention. Taxonomy of medication 
errors [Internet]. Available from: http://www.nccmerp.org/sites/default/files/taxonomy2001-0731.pdf

2. Bond CA, Raehl CL, Franke T. Clinical pharmacy services, hospital pharmacy staffing, and medication errors in United States hospitals. Pharmacotherapy. 2002;22(2):134-47.

3. Canadian Pharmacists Association. Blueprint for pharmacy: the vision for pharmacy. 2008 Jun; Available from: https://www.pharmacists.ca/cpha-ca/assets/File/pharmacy-incanada/blueprint/The\%20Vision\%20for\%20\%20Pharmacy_Apr\%201\%2009.pdf

4. The Society of Hospital Pharmacists of Australia (SHPA). Standards of practice for clinical pharmacy services. Soc Hosp Pharm Aust SHPA. 2005 J Pharm Pract Res ; 35(2;122-46.

5. Zellmer WA, Cobaugh DJ, Chen D. Three signals from the Pharmacy Practice Model Summit. Am J Health Syst Pharm. 2011 Jun 15;68(12):1077-1077.

6. Kohn LT, Corrigan J, Donaldson MS, editors. To err is human: building a safer health system. Washington, D.C: National Academy Press; 2000. 287 p.

7. Johnson JA, Bootman JL. Drug-related morbidity and mortality. A cost-of-illness model. Arch Intern Med. 1995 Oct 9;155(18):1949-56.

8. Pickette SG, Muncey L, Wham D. Implementation of a standard pharmacy clinical practice model in a multihospital system. Am J Health Syst Pharm. 2010 May 1;67(9):751-6.

9. Gallagher J, McCarthy S, Byrne S. Economic evaluations of clinical pharmacist interventions on hospital inpatients: a systematic review of recent literature. Int J Clin Pharm. 2014 Dec;36(6):110114.

10. Botero Aguirre JP, Valencia Quintero AF, Granados Vega EJ. Redesign of the Clinical Pharmacy Practice Model in a Tertiary Academic Hospital in Medellín, Colombia. Can J Hosp Pharm. 2017;70(3):225-31.

11. OMS | La OMS lanza una iniciativa mundial para reducir a la mitad los errores relacionados con la medicación en cinco años [Internet]. WHO. [cited 2017 May 7]. Available from: http://www.who.int/mediacentre/news/releases/2017/medication-related-errors/es/

12. Granados J, Salazar-Ospina A, Botero-Aguirre JP, Valencia-Quintero AF, Ortiz N, Amariles P. Effect and associated factors of a clinical pharmacy model in the incidence of medication errors (EACPharModel) in the Hospital Pablo Tobón Uribe: study protocol for a stepped wedge randomized controlled trial (NCT03338725). Trials. 2020 Jan 6;21(1):26.

13. Pastó-Cardona L, Masuet-Aumatell C, Bara-Oliván B, Castro-Cels I, Clopés-Estela A, Pàez-Vives F, et al. Estudio de incidencia de los errores de medicación en los procesos de utilización del medicamento: prescripción, transcripción, validación, preparación, dispensación y administración en el ámbito hospitalario. Farm Hosp. 2009 Sep;33(5):257-68.

14. Leguelinel-Blache G, Castelli C, Roux-Marson C, Bouvet S, Andrieu S, Cestac P, et al. Impact of collaborative pharmaceutical care on in-patients' medication safety: study protocol for a stepped wedge cluster randomized trial (MEDREV study). Trials. 2018;19(1):19. 
15. Khalili H, Farsaei S, Rezaee H, Dashti-Khavidaki S. Role of clinical pharmacists' interventions in detection and prevention of medication errors in a medical ward. Int J Clin Pharm. 2011;33(2):281-4.

16. Kuo GM, Touchette DR, Marinac JS. Drug errors and related interventions reported by United States clinical pharmacists: the American College of Clinical Pharmacy practice-based research network medication error detection, amelioration and prevention study. Pharmacotherapy. 2013;33(3):25365.

17. Wang T, Benedict N, Olsen KM, Luan R, Zhu X, Zhou N, et al. Effect of critical care pharmacist's intervention on medication errors: A systematic review and meta-analysis of observational studies. $J$ Crit Care. 2015;30(5):1101-6.

18. Basheti IA, Al-Qudah RA, Obeidat NM, Bulatova NR. Home medication management review in outpatients with chronic diseases in Jordan: a randomized control trial. Int J Clin Pharm. 2016;38(2):404-13.

19. Cullen DJ, Sweitzer BJ, Bates DW, Burdick E, Edmondson A, Leape LL. Preventable adverse drug events in hospitalized patients: A comparative study of intensive care and general care units. Crit Care Med. 1997;25(8):1289-97.

20. Kane-Gill S, Weber RJ. Principles and Practices of Medication Safety in the ICU. Crit Care Clin. 2006;22(2):273-90.

21. Tissot E, Cornette C, Demoly P, Jacquet M, Barale F, Capellier G. Medication errors at the administration stage in an intensive care unit. Intensive Care Med. 1999;25(4):353-9.

22. Rostami P, Power M, Harrison A, Bramfitt $K$, Williams SD, Jani $Y$, et al. Learning from the design, development and implementation of the Medication Safety Thermometer. Int J Qual Health Care J Int Soc Qual Health Care. 2017;29(2):301-9.

23. Garrouste-Orgeas M, Timsit JF, Vesin A, Schwebel C, Arnodo P, Lefrant JY, et al. Selected medical errors in the intensive care unit: results of the IATROREF study: parts I and II. Am J Respir Crit Care Med. 2010;181(2):134-42.

24. Löffler C, Drewelow E, Paschka SD, Frankenstein M, Eger J, Jatsch L, et al. Optimizing polypharmacy among elderly hospital patients with chronic diseases-study protocol of the cluster randomized controlled POLITE-RCT trial. Implement Sci. 2014;9(1):151.

25. Leufer T, Cleary-Holdforth J. Let's do no harm: medication errors in nursing: part 1. Nurse Educ Pract. 2013;13(3):213-6.

26. Bjornson DC, Hiner WO, Potyk RP, Nelson BA, Lombardo FA, Morton TA, et al. Effect of pharmacists on health care outcomes in hospitalized patients. Am J Hosp Pharm. 1993;50(9):1875-84.

27. Boyko WL, Yurkowski PJ, Ivey MF, Armitstead JA, Roberts BL. Pharmacist influence on economic and morbidity outcomes in a tertiary care teaching hospital. Am J Health-Syst Pharm AJHP Off J Am Soc Health-Syst Pharm. 1997;54(14):1591-5.

28. Haig GM, Kiser LA. Effect of pharmacist participation on a medical team on costs, charges, and length of stay. Am J Hosp Pharm. 1991;48(7):1457-62. 
29. Clapham CE, Hepler CD, Reinders TP, Lehman ME, Pesko L. Economic consequences of two drug-use control systems in a teaching hospital. Am J Hosp Pharm. 1988;45(11):2329-40.

30. Kucukarslan SN, Peters M, Mlynarek M, Nafziger DA. Pharmacists on rounding teams reduce preventable adverse drug events in hospital general medicine units. Arch Intern Med. 2003;163(17):2014-8.

31. Smythe MA, Shah PP, Spiteri TL, Lucarotti RL, Begle RL. Pharmaceutical Care in Medical Progressive Care Patients: Ann Pharmacother [Internet]. 2016 [cited 2020 Aug 25]; Available from: https://journals.sagepub.com/doi/10.1345/aph.17068

32. Pastó-Cardona L, Masuet-Aumatell C, Bara-Oliván B, Castro-Cels I, Clopés-Estela A, Pàez-Vives F, et al. Estudio de incidencia de los errores de medicación en los procesos de utilización del medicamento: prescripción, transcripción, validación, preparación, dispensación y administración en el ámbito hospitalario. Farm Hosp. 2009 Sep;33(5):257-68.

33. Machado Alba JE, Moreno Gutiérrez PA, Moncada Escobar JC. Errores de medicación identificados por un sistema de farmacovigilancia de instituciones hospitalarias en Colombia. Farm Hosp. 2015;39(6):338-49.

\section{Figures}




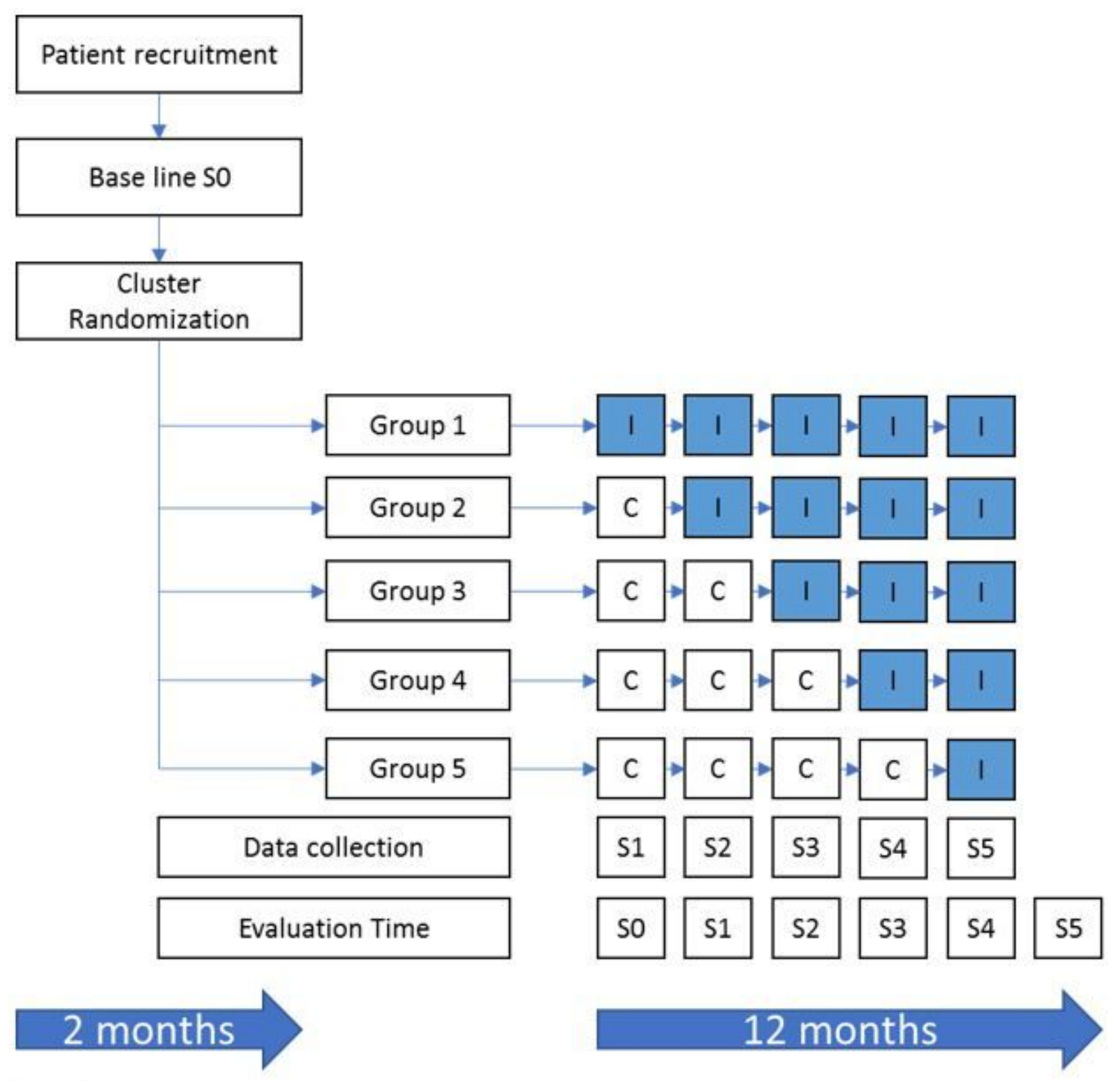

C Control: Common practice

1 Control: Clinical Pharmacy Model

Figure 1

Design of the EACPharModel study. This figure shows design and timeframe of the EACPharModel study. The outcomes to be measured during each evaluation stage are: incidence of medication error, time to the error and time to error recovery 


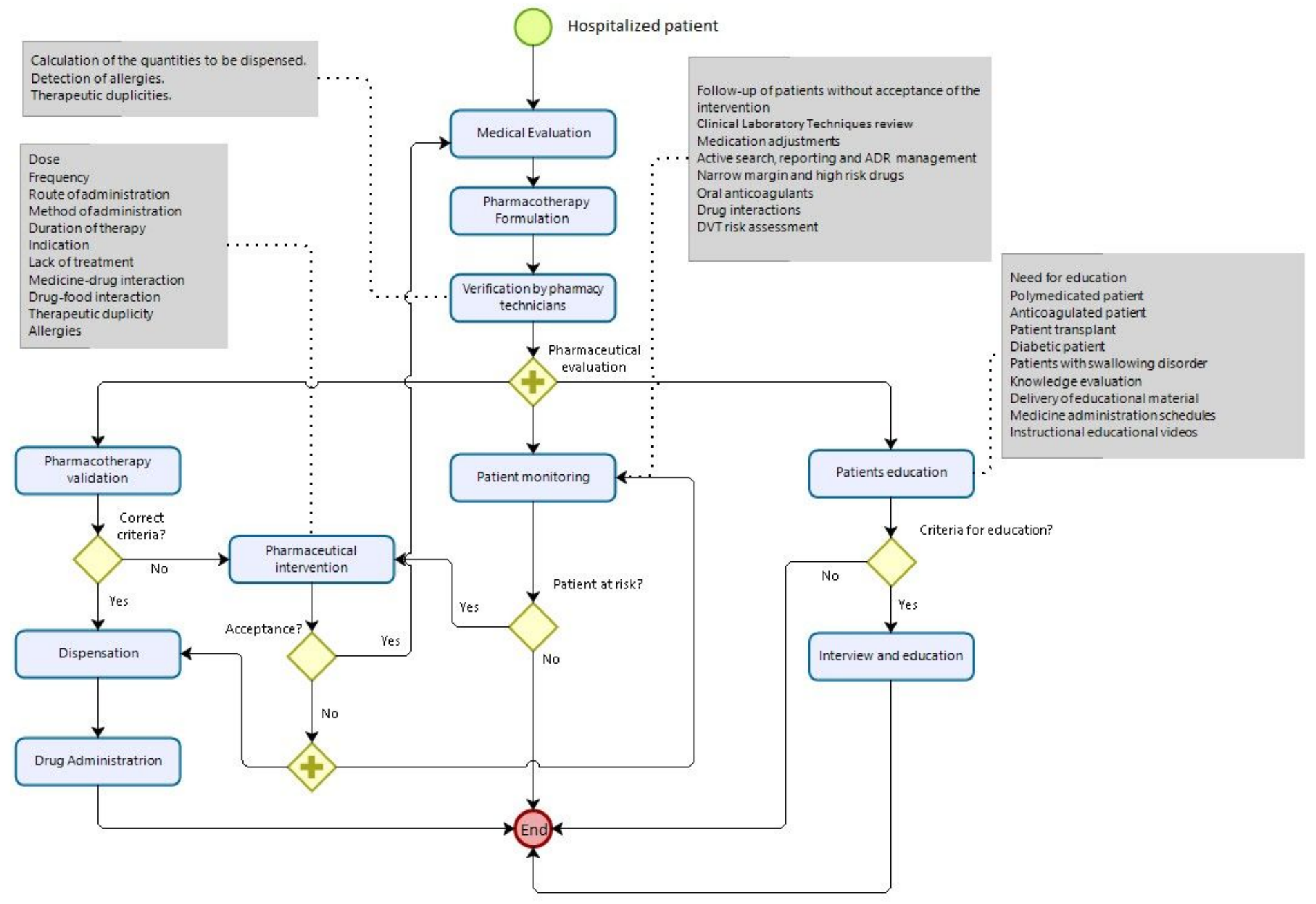

Figure 2

Clinical pharmacy practice model Hospital Pablo Tobón Uribe 
Calculation of the quantities to be dispensed.

Detection of allergies.

Therapeutic duplicities.

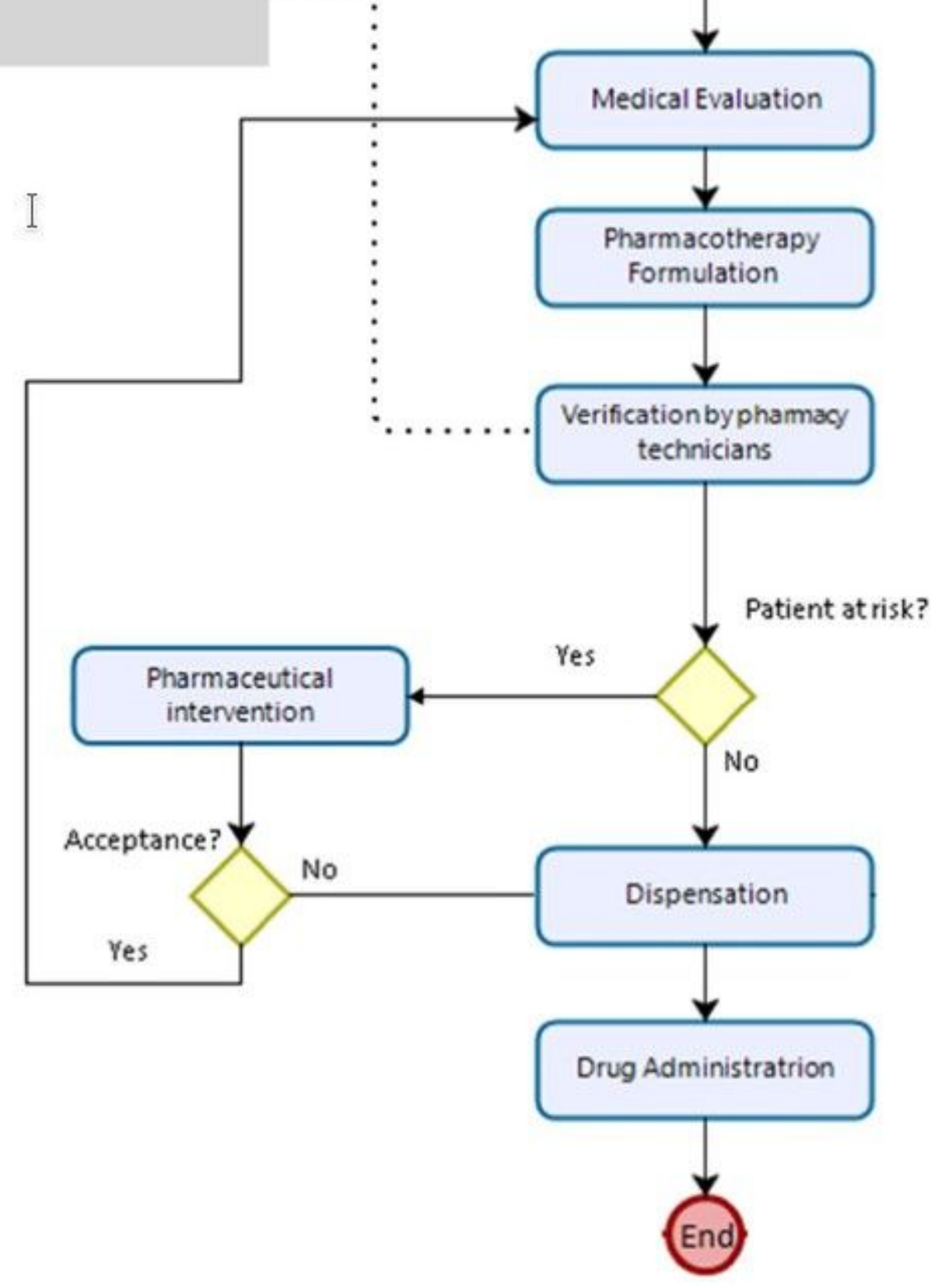

Figure 3

Usual care process in the Hospital Pablo Tobón Uribe 

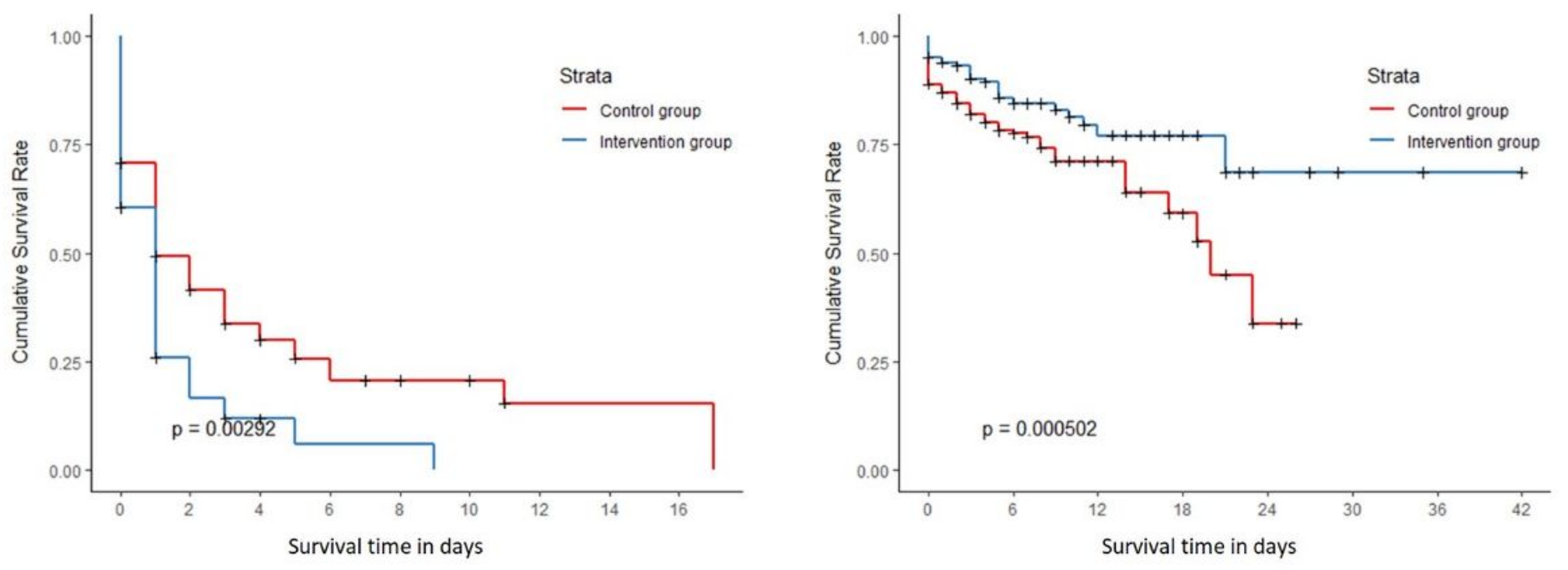

\section{Figure 4}

EACPharmodel study survival analysis. A) Time to a medication error, B) Time to recovery

\section{Supplementary Files}

This is a list of supplementary files associated with this preprint. Click to download.

- DB1.csv 